Research Paper

\title{
A Novel TNM Classification for Colorectal Cancers based on the Metro-ticket Paradigm
}

\author{
Jun-Peng Pei ${ }^{1 \#,}$, Chun-Dong Zhang1,2\#, Xiang Fu ${ }^{1}$, Yong Ba¹, Shuai Yue ${ }^{1}$, Zhe-Ming Zhao', Dong-Qiu Dai ${ }^{1,3 凶}$ \\ 1. Department of Gastrointestinal Surgery, The Fourth Affiliated Hospital of China Medical University, Shenyang 110032, China. \\ 2. Department of Gastrointestinal Surgery, Graduate School of Medicine, The University of Tokyo, Tokyo 113-0033, Japan. \\ 3. Cancer Center, The Fourth Affiliated Hospital of China Medical University, Shenyang 110032, China. \\ \#Co-first authors with equal contributions to this work.
}

$\square$ Corresponding author: Dong-Qiu Dai, E-mail: daidq63@163.com or cmudaidq@126.com; Tel.: +86-24-62043110; Fax: +86-24-62043110.

(c) The author(s). This is an open access article distributed under the terms of the Creative Commons Attribution License (https://creativecommons.org/licenses/by/4.0/). See http:/ /ivyspring.com/terms for full terms and conditions.

Received: 2020.10.27; Accepted: 2021.03.09; Published: 2021.04.05

\begin{abstract}
Background: Several revisions of the TNM classifications for colorectal cancer (CRC) have acknowledged that the oncological outcomes of stage IIB/IIC CRC are worse than those of stage IIIA. We aimed to develop a novel TNM (nTNM) classification based on the metro-ticket paradigm.

Methods: We identified eligible CRC patients from the Surveillance, Epidemiology, and End Results database. The nTNM was developed using distance from the origin on a Cartesian plane incorporating the $\mathrm{pN}$ (x-axis) and pT (y-axis) stages, and was compared with the AJCC TNM classification. The areas under the curves (AUCs), calibration curves, and Akaike's information criterion (AIC) were used to evaluate the predictive performances of the two classifications. Clinical benefits were further estimated by decision curve analyses. The validation cohort was applied to validate these findings.

Results: A total of 58,192 CRC patients (40,736 training cohort, 17,456 validation cohort) were finally included. In the training cohort, 18,476 patients (45.4\%) experienced upstaging and 15,907 patients (39.0\%) experienced downstaging in the nTNM classification compared with the TNM classification. Taking the prognosis of stage $I$ as the reference, survival decreased with increasing nTNM stage. The nTNM classification showed better discrimination (AUC, 0.678 vs. $0.667, P<0.001$ ), model-fitting (AIC, 236,525 vs. 237,741 ), and clinical benefits than the TNM classification. Similar results were found in the validation cohort.

Conclusions: The nTNM classification for CRC has better predictive performances and superior accuracy for predicting prognosis compared with the TNM classification. The nTNM classification should therefore be considered in future revisions of the TNM classification.
\end{abstract}

Key words: colorectal cancer, TNM classification, metro-ticket, novel TNM classification

\section{Introduction}

Colorectal cancer (CRC) is the third most commonly diagnosed cancer among both males and females in the United States [1]. The American Joint Committee on Cancer (AJCC) tumor-node-metastasis (TNM) staging system is the most widely used prognostic standard for CRC [2], and has been revised several times over the past few decades to improve its prognostic performance and treatment suggestions for patients with CRC. In general, the survival of patients with high-stage cancers should be lower than that of patients with low-stage cancers. However,
CRC may be an exception, especially with respect to stage IIB/C (T4N0) and stage IIIA (T1-2N1, T1N2a) tumors, with an apparent survival paradox between these stages. Based on the AJCC $7^{\text {th }}$ and $8^{\text {th }}$ TNM staging systems, data from the Surveillance, Epidemiology, and End Results (SEER) program indicated that the survival of patients with stage IIB/IIC CRC was worse than that of those with stage IIIA $[3,4]$. Similar results were also reported by the Japan Colon Cancer and Rectal Cancer Society and other research institutes [5-11]. 
Several recent studies have suggested a 'metroticket' paradigm to evaluate survival [12-16]. This paradigm was originally proposed by Mazzaferro et al. for hepatocellular cancer (HCC) and later evolved into a novel predictive tool for HCC patients after liver transplantation [12]. The metro-ticket paradigm was based on the concept that the longer the "trip" (i.e., increased $\mathrm{T}$ stage and $\mathrm{N}$ stage), the higher the price of the "ticket" (i.e. reduction in expected survival). Further research by $\mathrm{Lu}$ et al. showed that application of the metro-ticket system to the TNM staging system for gastric cancer could accurately stratify patients and improve its prognostic performance [15]. However, no studies have yet applied the metro-ticket paradigm to the TNM staging system for CRC.

We aimed to exploit a novel TNM (nTNM) staging system for CRC based on the metro-ticket system, compare the prognostic performances of the nTNM and the AJCC $8^{\text {th }}$ TNM staging systems, and validate these findings in a validation cohort.

\section{Methods}

\section{Patients}

Screened colorectal cancer patients from the SEER program from 1973 to 2015. The inclusion criteria were as follows: patients with CRC; provided the complete clinical information; patients were between 18 and 72 years old; first and single cancer; no distant metastasis (M0); performed surgical treatment; no neoadjuvant radiotherapy and chemotherapy were performed; survive more than 1 month; and follow-up at least 5 years or until death. The exclusion criteria were as follows: no complete clinical information; aged $<18$ or $>72$ years; have other malignant tumors; distant metastasis (M1); no surgical treatment; performed neoadjuvant radiotherapy and chemotherapy; survival time after surgery were less than 1 month; and follow-up time were less than 5 years or lost to follow-up.

\section{Development of $\mathbf{n T N M}$ staging system}

Based on the concept of the metro-ticket paradigm for gastric cancer, we proposed the current nTNM classification by combining the $\mathrm{pN}$ and $\mathrm{pT}$ stages, with preserved definitions of $\mathrm{pN}$ and $\mathrm{pT}$ stages. The nTNM was defined as the distance from the origin on a Cartesian plane that incorporated two variables: the $\mathrm{pN}$ stage (x-axis) and $\mathrm{pT}$ stage (y-axis); Pythagoras theorem was then used to calculate the distance of any given point from the origin of the plane $(0,0)$, where $\left[(n T N M)^{2}=(p N)^{2}+(p T)^{2}\right]$ (Figure 1A, 1B) [12-16]. The nTNM classification was similar to the AJCC classification and was divided into seven subgroups: I, IIA, IIB, IIC, IIIA, IIIB, and IIIC.

\section{Statistical analysis}

Categorical and continuous variables were described as frequency (\%) and median (interquartile range $[\mathrm{IQR}])$, respectively. The characteristics of the baseline clinical variables between the training and validation cohorts were compared with Student's $t$-test or $\chi^{2}$ test. Five-year overall survival (OS) and estimations of hazard ratios (HRs) were calculated and presented with $95 \%$ confidence intervals (CI). The prognostic performance of the nTNM was compared with that of the AJCC $8^{\text {th }}$ TNM classification. The Kaplan-Meier method with log-rank tests was applied. The model discrimination abilities of the nTNM and AJCC TNM staging systems were assessed by areas under the curves (AUCs), and the AUCs were compared by Hanley \& McNeil tests. Akaike's information criterion (AIC) was applied to evaluate the model-fitting [17]. Higher AUCs indicated better model discrimination, and lower values of AIC indicated superior model-fitting. Calibration curves were used to determine the agreement between the predicted and observed survival probabilities; in a perfect model, the predictions should fall on the diagonal $45^{\circ}$ line of the calibration curve [18]. Clinical benefit was further evaluated by decision curve analysis (DCA) [19].

All data were analyzed using MedCalc (Version 15.2, Ostend, Belgium), SPSS 22.0 (SPSS Inc., Chicago, IL, USA), and R version 3.6.1. All tests were two-sided and $P$ values $<0.05$ were considered statistically significant.

\section{Results}

\section{Patient characteristics}

Based on the inclusion and exclusion criteria, 58,192 patients with non-metastatic CRC were finally included and divided into a training $(\mathrm{n}=40,736)$ and a validation cohort $(\mathrm{n}=17,456)$ using a random sampling method (sampling ratio 7:3). The screening process is shown in Figure 1. In the training cohort, the median number of retrieved lymph nodes was 16.0 (IQR, 30.0-65.0). A total of 16,623 CRC patients had lymph node metastasis, with a median number of positive lymph nodes of 3.0 (IQR, 1.0-5.0). Tumor sizes were recorded for 37,033 patients, with a median of $4.0 \mathrm{~cm}$ (IQR, 3.0-6.0 cm). A total of 31,947 (78.4\%) patients had colon cancer and 8,789 (21.6\%) had rectal cancer. The clinical and pathological characteristics of the validation cohort were similar to those of the training cohort. Table 1 shows the detailed clinical and pathological characteristics of the training and validation cohorts. 


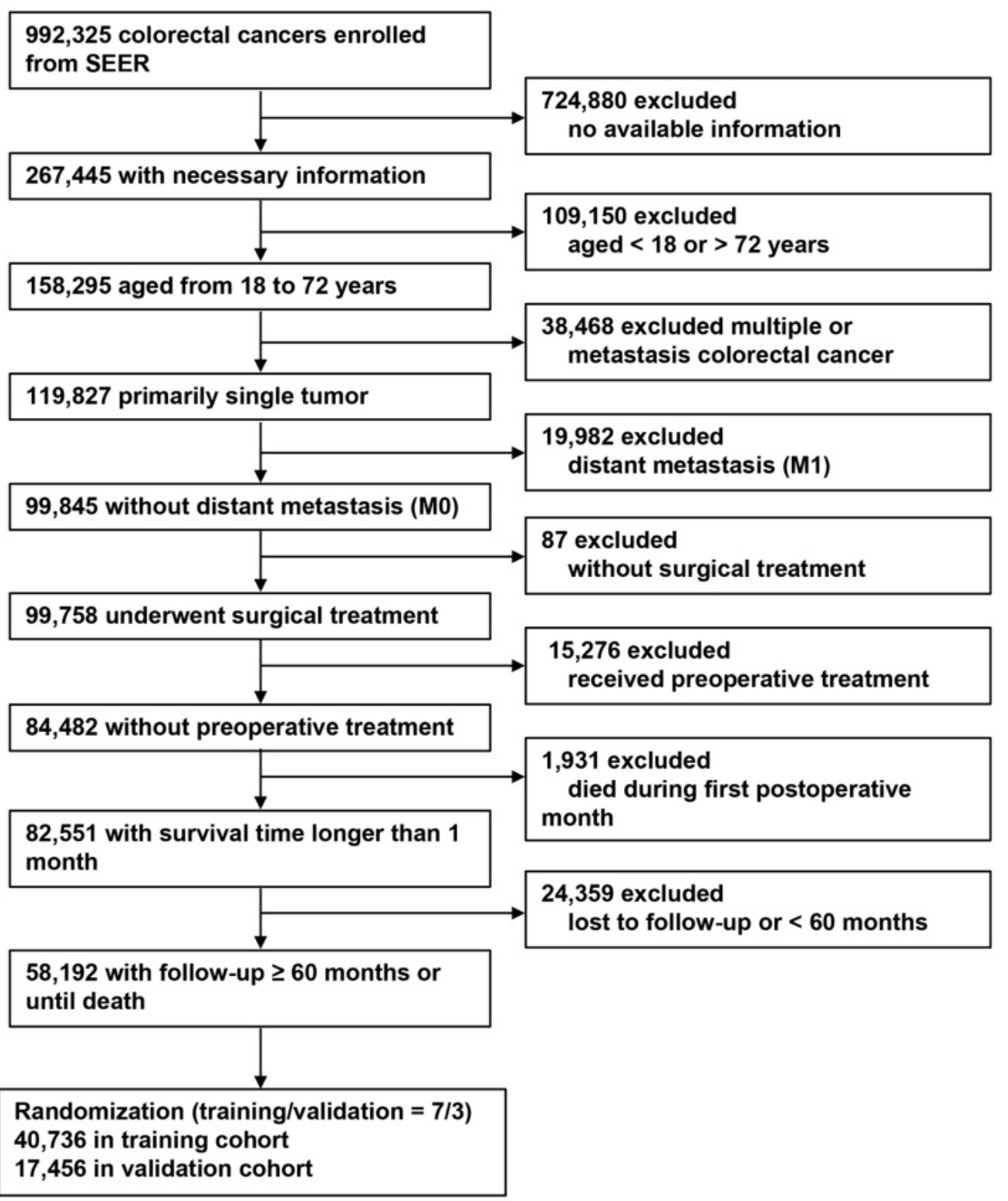

Figure 1. Flow chart for patient selection.

\section{The nTNM staging system}

The principle of the nTNM staging system involves using the distance from the origin on the Cartesian plane (Figure 2A, 2B). An intuitional comparison of the AJCC $8^{\text {th }}$ TNM and nTNM staging systems is shown in Figure 2C and 2D. The nTNM showed significant changes compared with the AJCC $8^{\text {th }}$ TNM staging system (Figure $2 \mathrm{E}$ ). In the training cohort, 18,476 patients (45.4\%) experienced upstaging in the TNM classification, including 5,397 patients $(13.2 \%)$ in stage I, 11,305 patients $(27.8 \%)$ in stage IIA, 925 patients $(2.3 \%)$ in stage IIB, and 849 patients $(2.1 \%)$ in stage IIC; while 15,907 patients $(39.0 \%)$ experienced downstaging, including 2,220 patients $(5.5 \%)$ in stage IIIA, 10,632 patients $(26.1 \%)$ in stage IIIB, and 3,025 patients (7.5\%) in stage IIIC (Figure 2E).

\section{OS based on AJCC TNM and nTNM staging systems}

According to AJCC $8^{\text {th }}$ TNM staging system, the
5 -year OS rates for each stage in the training cohort were: I, 91.7\%; IIA, 85.0\%; IIB, 73.0\%; IIC, 66.5\%; IIIA, $86.7 \%$; IIIB, $72.9 \%$; and IIIC, $47.9 \%$ (log-rank test, IIB vs IIIB: $P=0.875, P$ $<0.005$ for the other stages; Figure 3A). Kaplan-Meier analysis revealed a higher 5-year OS in patients with stage IIIA than in those with stages IIB and IIC (log-rank test, both $P<$ 0.001), higher 5-year OS in stage IIIB than stage IIC (log-rank test, $P<$ 0.001 ), and similar 5-year OS in stage IIB and stage IIIB (log-rank test, $P=$ $0.875)$. For the nTNM classification, the 5-year OS rates for each stage were: I, 93.2\%; IIA, 89.9\%; IIB, 83.7\%; IIC, 73.7\%; IIIA, 64.8\%; IIIB, 52.0\%; and IIIC, $34.7 \%$ (log-rank test, $P<$ 0.001 for all stages; Figure 3B). The 5-year OS rates increased sequentially from stages I to IIIC.

In the training cohort, the HRs of OS for each stage of the AJCC $8^{\text {th }}$ TNM staging systems were as follows: IIA, 1.665; IIB, 2.818; IIC, 3.540; IIIA, 1.364; IIIB, 2.839; and IIIC, 6.328 (stage I as the reference) (Table 2 and Figure 3). The HR for patients with stage IIIA was significantly lower than those for stages IIA, IIB, and IIC (all $P<$ $0.001)$, the HR for stage IIIB was higher than for stage IIC $(P<0.001)$, and the HRs for stage IIB stage IIIB were similar (log-rank test, $P=0.875$ ). HRs for each stage of the nTNM classification: IIA, 1.441; IIB, 2.151; IIC, 3.326; IIIA, 4.609; IIIB, 6.855; and IIIC, 11.385 (stage I as the reference). The HRs increased sequentially from stages I to IIIC. Similar results were also observed in patient groups with insufficient $(<12)$ and sufficient $(\geq 12)$ numbers of retrieved lymph nodes (Table S1 and Figure S1). Similar results were confirmed in the validation cohort (Table 2, Table S1, Figure 3C, 3D, and Figure S2).

\section{Comparison of prognostic performances between AJCC 8 ${ }^{\text {th }}$ TNM and nTNM staging systems}

The prognostic discrimination of the nTNM in the training cohort (AUC 0.678, 95\% confidence interval (CI), 0.673-0.682) was superior to that of the AJCC $8^{\text {th }}$ TNM classification (AUC 0.667, 95\% CI, 0.662-0.672) (Hanley \& McNeil test, $P<0.001$ ). The nTNM also had better model-fitting than the AJCC $8^{\text {th }}$ 
TNM classification (AIC, 236,525 vs. 237,741) (Table 3, Figure 4A). The calibration curves for 3-, and 5-year OS in the nTNM staging system also showed better agreement than the AJCC 8th TNM staging system (Figure 4C, 4D). Similar results were confirmed in the validation cohort (Table 3, Figure $4 \mathrm{~B}$ and Figure 4E, $4 \mathrm{~F})$.

Table 1. Clinical and pathological characteristics of the training and validation cohorts

\begin{tabular}{|c|c|c|c|c|c|c|c|}
\hline \multirow[t]{2}{*}{ Variable } & \multicolumn{2}{|c|}{$\begin{array}{l}\text { Univariate analysis } \\
\text { (training cohort) }\end{array}$} & \multirow[b]{2}{*}{$P$ value } & \multicolumn{2}{|c|}{$\begin{array}{l}\text { Univariate analysis } \\
\text { (validation cohort) }\end{array}$} & \multirow[b]{2}{*}{$P$ value } & \multirow{2}{*}{$\begin{array}{l}P \\
\text { value } \\
*\end{array}$} \\
\hline & $\begin{array}{l}\text { No. patients } \\
(\%)\end{array}$ & $\begin{array}{l}5- \\
\text { year } \\
\text { OS }\end{array}$ & & $\begin{array}{l}\text { No. patients } \\
(\%)\end{array}$ & $\begin{array}{l}5 \text {-year } \\
\text { OS }\end{array}$ & & \\
\hline Age, year & & & $<0.001$ & & & $<0.001$ & 0.281 \\
\hline$\leq 60$ & 21747 (53.4) & $82.6 \%$ & & 9234 (52.9) & $83.1 \%$ & & \\
\hline$>60$ & 18989 (46.6) & $75.8 \%$ & & $8222(47.1)$ & $75.0 \%$ & & \\
\hline Gender & & & $<0.001$ & & & $<0.001$ & 0.381 \\
\hline Female & 18807 (46.2) & $81.4 \%$ & & 8128 (46.6) & $81.2 \%$ & & \\
\hline Male & $21929(53.8)$ & $77.7 \%$ & & $9328(53.4)$ & $77.7 \%$ & & \\
\hline Race & & & $<0.001$ & & & $<0.001$ & 0.632 \\
\hline White & 31150 (76.5) & $80.2 \%$ & & $13360(76.5)$ & $80.0 \%$ & & \\
\hline Black & $5560(13.6)$ & $72.4 \%$ & & $2408(13.8)$ & $72.6 \%$ & & \\
\hline Other & $4026(9.9)$ & $82.9 \%$ & & $1688(9.7)$ & $83.5 \%$ & & \\
\hline Location & & & 0.895 & & & 0.808 & 0.228 \\
\hline Colon & 31947 (78.4) & $79.2 \%$ & & $13611(78.0)$ & $79.2 \%$ & & \\
\hline Rectum & 8789 (21.6) & $80.2 \%$ & & $3845(22.0)$ & $79.6 \%$ & & \\
\hline Size, $\mathrm{cm}$ & & & $<0.001$ & & & $<0.001$ & 0.083 \\
\hline$\leq 4$ & $18747(46.0)$ & $82.4 \%$ & & 8064 (46.2) & $82.1 \%$ & & \\
\hline$>4$ & $18286(44.9)$ & $75.3 \%$ & & 7720 (44.2) & $75.3 \%$ & & \\
\hline Unknown & $3703(9.1)$ & $84.8 \%$ & & $1672(9.6)$ & $84.2 \%$ & & \\
\hline \multicolumn{2}{|c|}{ Histological grade } & & $<0.001$ & & & $<0.001$ & 0.236 \\
\hline Grade I & $3877(9.5)$ & $86.9 \%$ & & $1620(9.3)$ & $86.6 \%$ & & \\
\hline Grade II & 29898 (73.4) & $80.7 \%$ & & $12812(73.4)$ & $80.9 \%$ & & \\
\hline Grade III & $6335(15.6)$ & $69.8 \%$ & & $2735(15.7)$ & $69.3 \%$ & & \\
\hline Grade IV & $626(1.5)$ & $67.2 \%$ & & $289(1.7)$ & $64.0 \%$ & & \\
\hline $\begin{array}{l}\text { AJCC } 8^{\text {th }} \\
\text { pT stage }\end{array}$ & & & $<0.001$ & & & $<0.001$ & 0.348 \\
\hline $\mathrm{T} 1$ & $6267(15.4)$ & $92.4 \%$ & & 2733 (15.7) & $91.0 \%$ & & \\
\hline $\mathrm{T} 2$ & 7171 (17.6) & $88.8 \%$ & & $2970(17.0)$ & $88.8 \%$ & & \\
\hline $\mathrm{T} 3$ & $22826(56.0)$ & $77.2 \%$ & & $9919(56.8)$ & $77.5 \%$ & & \\
\hline T4a & $2579(6.3)$ & $61.6 \%$ & & $1038(5.9)$ & $60.7 \%$ & & \\
\hline $\mathrm{T} 4 \mathrm{~b}$ & $1893(4.6)$ & $51.5 \%$ & & $796(4.6)$ & $49.6 \%$ & & \\
\hline \multicolumn{2}{|c|}{ AJCC $8^{\text {th }} \mathrm{pN}$ stage } & & $<0.001$ & & & $<0.001$ & 0.009 \\
\hline No & $23889(58.6)$ & $86.9 \%$ & & $10380(59.5)$ & $86.1 \%$ & & \\
\hline $\mathrm{N} 1 \mathrm{a}$ & $4978(12.2)$ & $78.9 \%$ & & $2182(12.5)$ & $79.1 \%$ & & \\
\hline $\mathrm{N} 1 \mathrm{~b}$ & 5499 (13.5) & $73.4 \%$ & & $2248(12.9)$ & $72.8 \%$ & & \\
\hline $\mathrm{N} 2 \mathrm{a}$ & $3416(8.4)$ & $63.5 \%$ & & $1495(8.6)$ & $65.6 \%$ & & \\
\hline $\mathrm{N} 2 \mathrm{~b}$ & $2954(7.3)$ & $49.1 \%$ & & $1151(6.6)$ & $49.0 \%$ & & \\
\hline \multicolumn{2}{|c|}{ AJCC $8^{\text {th }}$ TNM stage } & & $<0.001$ & & & $<0.001$ & 0.063 \\
\hline I & $10810(26.5)$ & $91.7 \%$ & & $4585(26.3)$ & $90.8 \%$ & & \\
\hline IIA & $11305(27.8)$ & $85.0 \%$ & & $5046(28.9)$ & $84.9 \%$ & & \\
\hline IIB & $925(2.3)$ & $73.0 \%$ & & $365(2.1)$ & $70.8 \%$ & & \\
\hline IIC & $849(2.1)$ & $66.5 \%$ & & $384(2.2)$ & $60.1 \%$ & & \\
\hline IIIA & $2223(5.5)$ & $86.7 \%$ & & $972(5.6)$ & $87.2 \%$ & & \\
\hline IIIB & $10632(26.1)$ & $72.9 \%$ & & $4526(25.9)$ & $73.0 \%$ & & \\
\hline IIIC & $3992(9.8)$ & $47.9 \%$ & & $1578(9.0)$ & $47.8 \%$ & & \\
\hline \multicolumn{2}{|c|}{ No. of rLNs } & & & & & $<0.001$ & \\
\hline$<12$ & $10548(25.9)$ & $77.5 \%$ & & $4516(25.9)$ & $76.4 \%$ & & \\
\hline$\geq 12$ & $30188(74.1)$ & $80.1 \%$ & & $12940(74.1)$ & $80.3 \%$ & & \\
\hline
\end{tabular}

AJCC, No., number; OS, overall survival; pT stage, pathological T stage; $\mathrm{pN}$ stage, pathological $\mathrm{N}$ stage; rLNs, retrieved lymph nodes.

*P values of log-rank tests.

\section{Clinical usefulness}

We evaluated the clinical usefulness of the AJCC $8^{\text {th }}$ TNM and nTNM staging systems in the training and validation cohorts by DCAs. In the training and validation cohorts, the net benefit of the nTNM staging system was higher than that of the AJCC 8th TNM staging system between threshold probabilities of around $10 \%-20 \%$ in predicting 3-year OS, and between threshold probabilities of around $20 \%-35 \%$ in predicting 5-year OS (Figure 5).

Table 2. Three- and five-year OS and $95 \% \mathrm{Cl}$ for AJCC 8th TNM classification and nTNM classification in training and validation cohorts

\begin{tabular}{|c|c|c|c|c|c|}
\hline Variable & No. of patients (\%) & HR $(95 \% \mathrm{CI})$ & 3-year OS & 5-year OS & $P$ value \\
\hline \multicolumn{6}{|c|}{ Training cohort } \\
\hline \multicolumn{5}{|c|}{ TNM stage } & $<0.001$ \\
\hline I & $10692(26.2)$ & 1 (Reference) & $95.1 \%$ & $91.7 \%$ & \\
\hline IIA & $11451(28.1)$ & $1.67(1.57-1.77)$ & $90.7 \%$ & $85.0 \%$ & \\
\hline IIB & $911(2.2)$ & $2.82(2.50-3.17)$ & $82.0 \%$ & $73.0 \%$ & \\
\hline IIC & $858(2.1)$ & $3.54(3.16-3.97)$ & $75.6 \%$ & $66.5 \%$ & \\
\hline IIIA & $2202(5.4)$ & $1.36(1.23-1.52)$ & $92.1 \%$ & $86.7 \%$ & \\
\hline IIIB & $10679(26.2)$ & $2.84(2.68-3.01)$ & $83.0 \%$ & $72.9 \%$ & \\
\hline IIIC & $3943(9.7)$ & $6.33(5.94-6.74)$ & $60.8 \%$ & $47.9 \%$ & \\
\hline \multicolumn{5}{|c|}{ nTNM staging system } & $<0.001$ \\
\hline I & $9449(23.2)$ & 1 (Reference) & $96.0 \%$ & $93.2 \%$ & \\
\hline IIA & $11647(28.6)$ & $1.44(1.31-1.59)$ & $94.0 \%$ & $89.9 \%$ & \\
\hline IIB & $6461(15.9)$ & $2.15(1.98-2.34)$ & $90.1 \%$ & $83.7 \%$ & \\
\hline IIC & $5729(14.1)$ & $3.33(3.05-3.63)$ & $83.4 \%$ & $73.7 \%$ & \\
\hline IIIA & 5410 (13.3) & $4.61(4.22-5.04)$ & $76.7 \%$ & $64.8 \%$ & \\
\hline IIIB & $920(2.3)$ & $6.86(6.26-7.5)$ & $65.1 \%$ & $52.0 \%$ & \\
\hline IIIC & $1120(2.7)$ & $11.4(10.2-12.7)$ & $46.4 \%$ & $34.7 \%$ & \\
\hline \multicolumn{6}{|c|}{ Validation cohort } \\
\hline \multicolumn{5}{|c|}{ TNM classification } & $<0.001$ \\
\hline I & $10692(26.2)$ & 1 (Reference) & $95.0 \%$ & $90.8 \%$ & \\
\hline IIA & $11451(28.1)$ & $1.60(1.46-1.76)$ & $91.4 \%$ & $84.9 \%$ & \\
\hline IIB & $911(2.2)$ & $3.14(2.62-3.77)$ & $78.2 \%$ & $70.8 \%$ & \\
\hline IIC & $858(2.1)$ & $4.22(3.58-4.97)$ & $70.9 \%$ & $60.1 \%$ & \\
\hline IIIA & $2202(5.4)$ & $1.43(1.23-1.68)$ & $93.0 \%$ & $87.2 \%$ & \\
\hline IIIB & $10679(26.2)$ & $2.77(2.53-3.02)$ & $83.2 \%$ & $73.0 \%$ & \\
\hline IIIC & $3943(9.7)$ & $5.99(5.43-6.60)$ & $61.8 \%$ & $47.8 \%$ & \\
\hline \multicolumn{5}{|c|}{ nTNM classification } & $<0.001$ \\
\hline I & $9449(23.2)$ & 1 (Reference) & $95.5 \%$ & $91.6 \%$ & \\
\hline IIA & 11647 (28.6) & $1.34(1.16-1.54)$ & $94.2 \%$ & $89.8 \%$ & \\
\hline IIB & $6461(15.9)$ & $1.96(1.74-2.22)$ & $90.6 \%$ & $83.7 \%$ & \\
\hline IIC & 5729 (14.1) & $3.38(2.96-3.85)$ & $82.4 \%$ & $72.4 \%$ & \\
\hline IIIA & 5410 (13.3) & $4.26(3.74-4.86)$ & $76.7 \%$ & $64.4 \%$ & \\
\hline IIIB & $920(2.3)$ & $6.14(5.35-7.04)$ & $66.2 \%$ & $51.9 \%$ & \\
\hline IIIC & $1120(2.7)$ & $9.98(8.45-11.8)$ & $48.2 \%$ & $35.8 \%$ & \\
\hline
\end{tabular}

$\mathrm{CI}$, confidence interval; HR, hazard ratio; OS, overall survival; No., number; nTNM, novel TNM; TNM, tumor-node-metastasis.

Table 3. Prognostic performances of AJCC 8th TNM classification and nTNM classification in training and validation cohorts

\begin{tabular}{lccc}
\hline Variables & AUC $(95 \% \mathrm{CI})$ & AIC & $P$ value \\
\hline Training cohort & & & $<0.001$ \\
TNM classification & $0.667(0.662-0.672)$ & 237741 & \\
nTNM classification & $0.678(0.673-0.682)$ & 236525 & \\
Validation cohort & & & $<0.001$ \\
TNM classification & $0.660(0.653-0.667)$ & 92884 & \\
nTNM classification & $0.671(0.664-0.678)$ & 92414 & \\
AUC, the areas under the curve; AIC, Akaike's information criterion; CI, confidence \\
interval; nTNM, novel TNM; TNM, tumor-node-metastasis. & \\
*Hanley \& McNeil tests of AUCs.
\end{tabular}




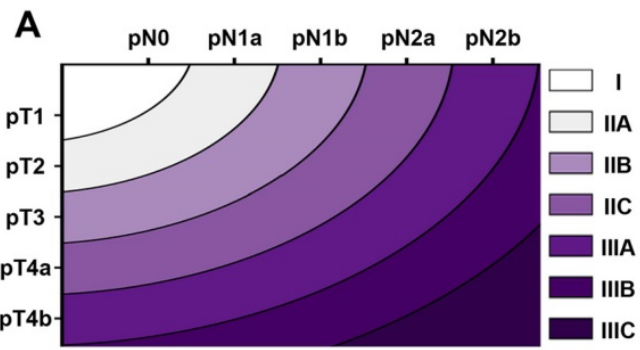

B

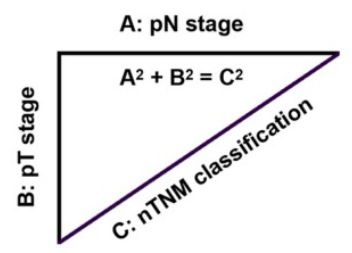

C

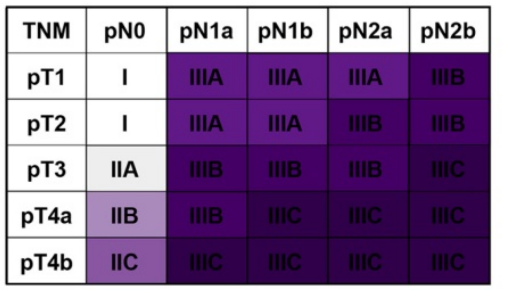

D

E

No. of patients in novel TNM (nTNM) classification

\begin{tabular}{|c|c|c|c|c|c|c|c|}
\hline & I & IIA & IIB & IIC & IIIA & IIIB & IIIC \\
\hline 1 & 5413 & 5397 & 0 & 0 & 0 & 0 & 0 \\
\hline \multirow{3}{*}{ 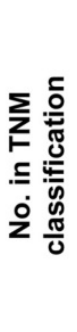 } & 0 & 0 & 11305 & 0 & 0 & 0 & 0 \\
\hline & 0 & 0 & 0 & 925 & 0 & 0 & 0 \\
\hline & 0 & 0 & 0 & 0 & 849 & 0 & 0 \\
\hline z & 0 & 1206 & 947 & 70 & 0 & 0 & 0 \\
\hline IIIB & 0 & 0 & 3188 & 4396 & 3048 & 0 & 0 \\
\hline IIIC & 0 & 0 & 0 & 0 & 255 & 2797 & 940 \\
\hline
\end{tabular}

Upstaging

Downstaging

Figure 2. A: the novel TNM (nTNM) classification was defined as the distance from the origin on a Cartesian plane that incorporated two variables: the $\mathrm{pN}$ stage (x-axis) and $\mathrm{pT}$ stage (y-axis); $\mathrm{B}$ : the Pythagoras theorem was used to calculate the distance of any given point from the origin of the plane $(0$, $0)$, where $\left[(n T N M) 2=(p N)^{2}+(p T)^{2}\right]$; C: AJCC $8^{\text {th }}$ TNM classification; D: nTNM classification; E: the staging migration between the nTNM and AJCC 8th TNM staging systems in the training cohort.
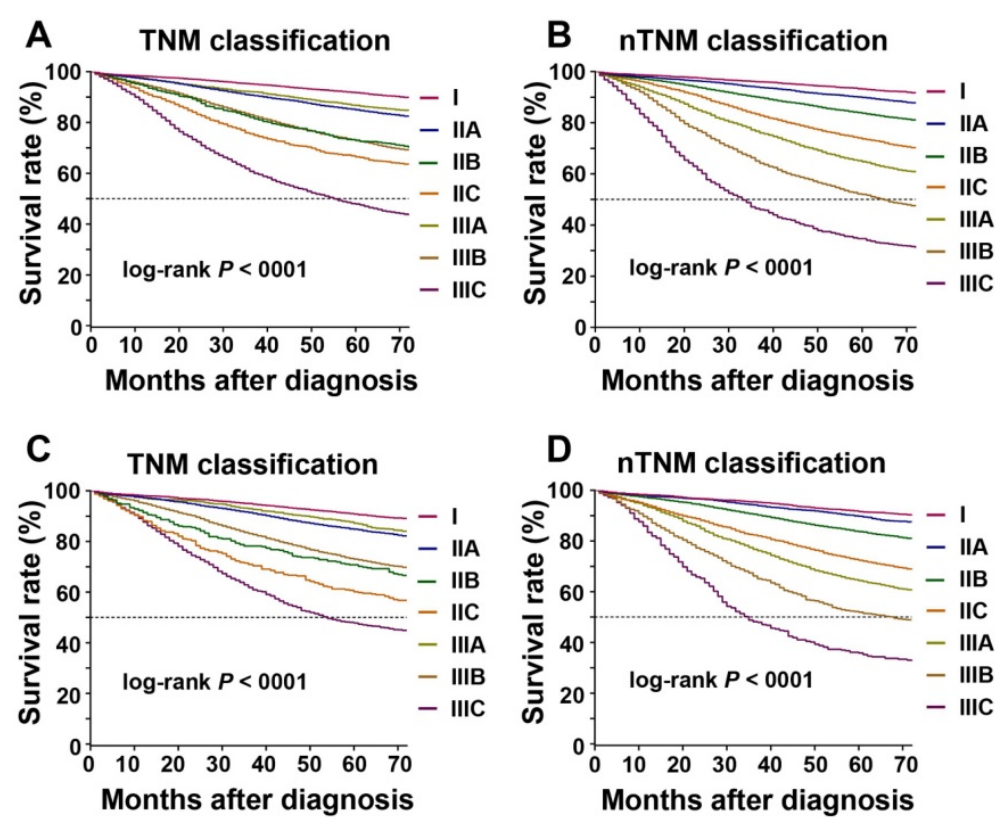

Figure 3. Kaplan-Meier survival curve for overall survival according to the AJCC $8^{\text {th }}$ TNM and novel TNM (nTNM) staging systems. A: the AJCC 8th TNM staging system in the training cohort; $B$ : the nTNM staging system in the training cohort; C: the AJCC $8^{\text {th }}$ TNM staging system in the validation cohort; D: the nTNM staging system in the validation cohort.

\section{Discussion}

Accurate cancer staging helps doctors to predict survival and provide more effective therapeutic recommendations. According to the AJCC TNM classifications, the survival of patients with low-stage cancers is generally higher than that for high-stage cancers among patients with most solid malignant tumors [20]. However, CRC is one of the few exceptions, and many studies have shown an obvious survival paradox between patients with stage IIB/IIC and stage IIIA CRC [6,8-11]. The current study confirmed that the survival of patients with stage IIIA CRC was lower than that of patients with stage IIB/IIC CRC.

Researchers have proposed numerous explanations for this paradox, including possible staging migration as a result of retrieval of insufficient lymph nodes [21]. This means that the survival of patients with stage IIB/C disease who undergo sufficient lymphadenectomy would be better than that of patients with stage IIIA CRC. However, previous studies showed that the survival paradox persisted, even if sufficient lymph nodes were retrieved [6,9]. Some researchers also attributed the survival paradox to preferential receipt of adjuvant radiotherapy and chemotherapy in stage IIIA disease or a lack of systemic treatment in stage IIB/C CRC $[21,22]$. The role of postoperative adjuvant chemotherapy in patients with stage II CRC remains controversial. The American Society of Clinical Oncology recommends postoperative adjuvant chemotherapy for patients with stage $\mathrm{T} 4$ CRC [23], while several studies have shown that patients with stage IIB/C CRC who receive adjuvant chemotherapy still have significantly lower survival than stage IIIA patients $[6,9,11]$. An inadequate use of postoperative chemotherapy therefore cannot explain the poor prognosis of patients with stage IIB/C CRC. Another possibility is that the poor prognosis of stage IIB/C CRC may be related to the presence of residual tumor after resection $[6,7,24]$. Negative margins can be achieved more easily for T1-T2 lesions (i.e., stage IIIA), while extensive 
en-bloc resection should be performed for T4 lesions (i.e., stage IIB/C), but such surgery is more complicated. Resection of stage IIB/C tumors is thus more likely to result in positive margins than resection of stage IIIA tumors. Nonetheless, among patients with positive margins, the survival of patients with stage IIB/C CRC was still lower than that of stage IIIA, and the survival of patients with stage IIB/C and negative margins was similar to that of patients with stage IIIA and positive margins $[6,7]$.

\section{A}

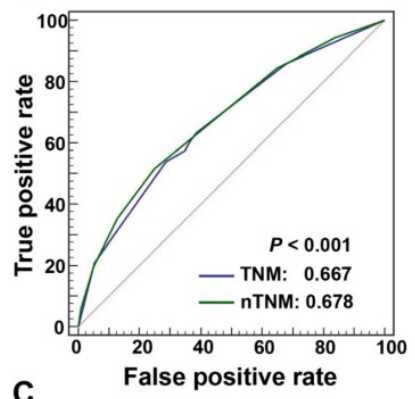

B
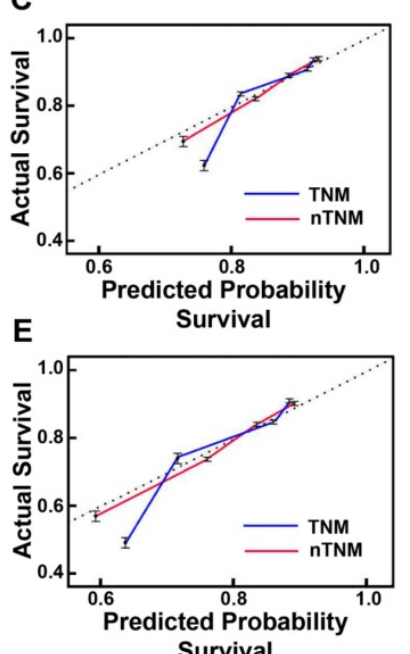

Survival

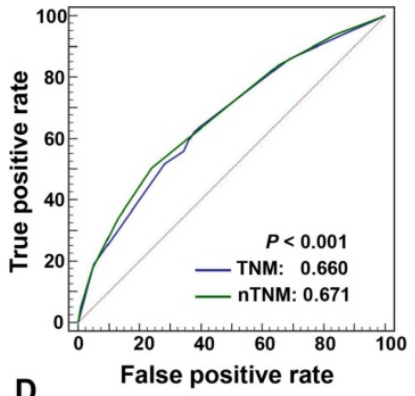

D

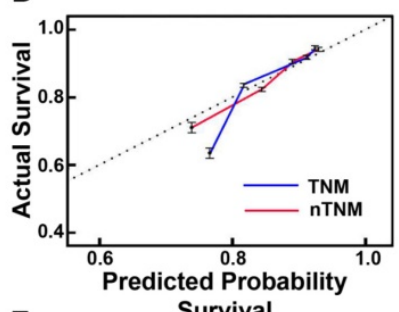

$\mathbf{F}$

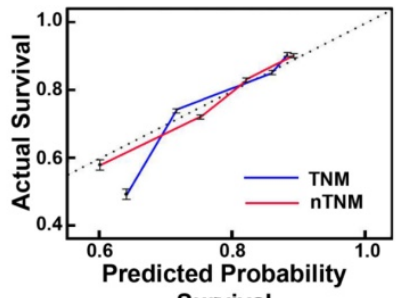

Survival

Figure 4. The areas under the curves (AUCs) and calibration curves for predicting patient survival. A: AUCs of the AJCC $8^{\text {th }}$ TNM staging system and novel TNM (nTNM) staging system in the training cohort; B: AUCs of the AJCC 8th TNM staging system and nTNM staging system in the validation cohort; $C$, At three-year overall survival (OS) in the training cohort; D, At three-year OS in the validation cohort; $E$, At five-year OS in the training cohort; F, At five-year OS in the validation cohort.

This paradox is widely thought to be caused by the inherent biological invasiveness of stage IIB/C CRC, with various lines of evidence to support this view. Perineural invasion, a high-frequency of microsatellite instability and the tumor's inflammatory reaction can all increase the biological aggressiveness of the tumor. Previous studies showed that stage IIB/C CRC had a higher proportion of perineural invasion, a high frequency of microsatellite instability, and a more prominent protumor inflammatory reaction compared with stage IIIC CRC $[9,11]$. Previous studies have shown characteristic differences between stages IIB/C and IIIA, with stage

IIB/IIC cancers showing faster intestinal wall infiltration and slower lymph node metastasis, and stage IIIA cancers having slower intestinal wall infiltration and earlier lymphatic infiltration $[9,11]$. This biological difference suggests that early stages do not translate into better oncological outcomes or less recurrence. Some researchers used gene expression profiling to stratify colon cancer according to the risk of recurrence, and indicated that stage IIA/C CRC had poor tumor prognosis and high recurrence rates $[25,26]$.
A

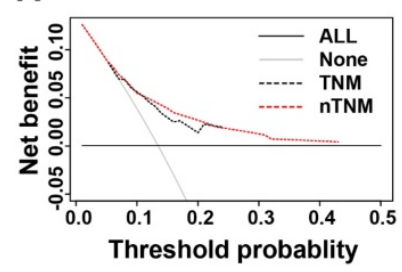

C

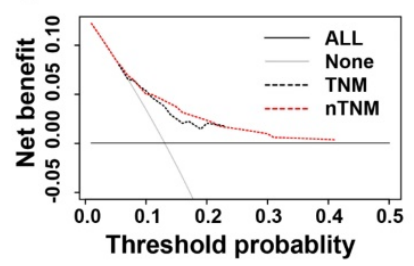

B

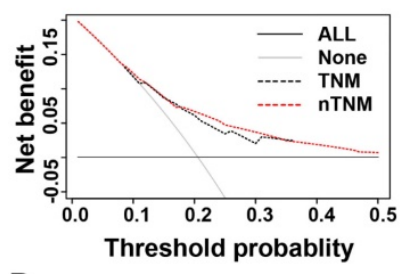

D

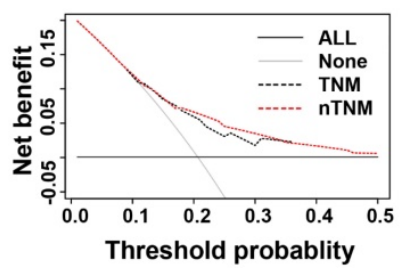

Figure 5. Decision curve analysis (DCA) of three- and five-year overall survival (OS) of novel TNM (nTNM) staging system and AJCC $8^{\text {th }}$ TNM staging system. A: the three-year OS in the training cohort; B: the five-year OS in the training cohort; C: the three-year OS in the validation cohort; D: the five-year OS in the validation cohort.

$\mathrm{Li}$ et al. suggested that the survival paradox between stages IIB/IIC and IIIA CRC was derived from the inherent concept of the current TNM classification that lymph node metastases ( $\mathrm{N}$ stage) affect the prognosis more significantly than local invasion (T stage) $[27,28]$. In the past few decades, the AJCC system has undergone several revisions, and compared with the AJCC $6^{\text {th }}$ TNM classification for $\mathrm{CRC}$, the $7^{\text {th }} \mathrm{TNM}$ classification increased the weight of T stage. Patients with CRC that directly infiltrates or adheres to adjacent organs or structures have poor outcomes. The AJCC $7^{\text {th }}$ TNM classification therefore divides stage T4 into stage T4a (tumor penetrates the visceral peritoneal surface) and stage T4b (tumor directly invades or histologically adheres to adjacent organs or structures). T4bN0 lesions were reclassified from stage IIB to stage IIC and T1-2N2 lesions from IIIC to IIIB/IIIC $[4,29]$. These modifications remained unchanged in the latest AJCC 8th TNM classification", and mirrored the increased weighting of $\mathrm{T}$ stage in the TNM classification for CRC. However, $\mathrm{Li}$ et al. indicated that the current TNM classification still underestimates the weight of $\mathrm{T}$ stage and overestimates the weight of $\mathrm{N}$ stage $[27,28]$. The above research showed that the survival paradox between 
IIB/IIC and IIIA CRC was essentially related to the inherent biological invasiveness of stage IIB/C, and the fact that the AJCC $8^{\text {th }}$ TNM classification underestimates the weight of $\mathrm{T}$ stage or overestimates the weight of $\mathrm{N}$ stage. We therefore proposed the nTNM classification based on the metro-ticket paradigm to resolve this survival paradox.

The metro-ticket paradigm was originally used to predict the long-term prognosis of patients undergoing liver transplantation for $\mathrm{HCC}^{12}$, and this prognostic model was shown to accurately stratify the survival of HCC patients. This theory was subsequently applied to patients with CRC liver metastases, thus establishing a novel tumor burden scoring system that could accurately predict patient prognosis after resection in patients with CRC liver metastases ${ }^{14}$. In another study, Lu et al. developed a new TNM gastric cancer classification based on the same theory and showed that this new system was superior to the AJCC $8^{\text {th }}$ TNM classification for predicting the long-term survival of patients with gastric cancer ${ }^{15}$. However, no study has yet developed a novel TNM classification for CRC based on the same theory. We therefore modeled $\mathrm{pT}$ and $\mathrm{pN}$ stages on a Cartesian plane and predicted the survival of CRC patients based on this model. Applying the principle of the metro-ticket paradigm, the weights of the $\mathrm{pT}$ and $\mathrm{pN}$ stages were considered equally.

Rice et al. summarized the characteristics of an excellent classification as: distinguishability between different stages, homogeneity in the same stage, and monotonicity of gradients [30]. Regarding distinguishability, the nTNM showed significant differences in survival between each pair of stages (log-rank test, all $P<0.001$ ), whereas the AJCC $8^{\text {th }}$ TNM classification showed no significant difference in survival between stages IIB and IIIB (log-rank test, $P=0.875)$. The nTNM classification also performed better than the AJCC $8^{\text {th }}$ TNM classification in terms of gradient monotonicity. The Kaplan-Meier survival curves indicated that the nTNM classification distinguished patients into different stages, with decreasing survival corresponding with increased stage; in contrast, the survival of stage IIB/C was higher than that of stage IIIA according to the AJCC $8^{\text {th }}$ TNM classification.

The nTNM also showed significantly better ability to predict prognosis and clinical utility than the AJCC $8^{\text {th }}$ TNM classification. In this study, the nTNM classification had a lower AIC value and a higher AUC value (Hanley \& McNeil test, all $P<0.001$ ) than the AJCC $8^{\text {th }}$ TNM classification. The calibration curve of survival probability also manifested better consistency between predicted and observed survival in the nTNM staging system. DCA analysis demonstrated that the nTNM staging system had better clinical benefits than the AJCC $8^{\text {th }}$ TNM staging system.

The large sample size of this study suggests that the results were reliable. However, the study also had some limitations. First, it was a retrospective study and may therefore have been affected by selection bias and unknown confounding factors. Further prospective studies and other large-scale multicenter data are therefore needed to verify our results. In addition, postoperative adjuvant therapy may affect the prognosis of patients with CRC; however, SEER data lacks information on adjuvant therapy, which may have had an impact on the prognostic ability of the TNM classification.

\section{Conclusions}

The nTNM staging system has better prognostic performance than the AJCC TNM $8^{\text {th }}$ staging system for predicting the prognosis of patients with CRC. We therefore suggest that the nTNM staging system should be considered in future revisions of the AJCC TNM staging system. However, the findings of the current study require further external validation.

\section{Abbreviations}

AJCC: American Joint Committee on Cancer; AIC: Akaike's information criterion; AUC: the areas under the curve; $\mathrm{CI}$ : confidence interval; $\mathrm{CRC}$ : colorectal cancer; DCA: decision curve analysis; HCC: hepatocellular cancer; HR: hazard ratio; IQR: interquartile range; OS: overall survival; SEER: Surveillance, Epidemiology, and End Results; TNM: tumor-node-metastasis.

\section{Supplementary Material}

Supplementary figures and tables. http://www.jcancer.org/v12p3299s1.pdf

\section{Acknowledgements}

The authors acknowledge the efforts of the Surveillance, Epidemiology, and End Results (SEER) Program tumor registries for the support (https://seer.cancer.gov/). This research was funded by the China Scholarship Council, grant number 201908050148.

\section{Funding Support}

This research was funded by the China Scholarship Council, grant number 201908050148.

\section{Data availability statement}

The datasets analyzed during this current study are available in SEER database (https://seer.cancer. gov/) to extract the eligible cases. The data are also 
available from the corresponding author on reasonable request.

\section{Author Contributions}

J.-P. P. and C.-D. Z. wrote the main text and performed data analysis. J.-P. P., C.-D. Z. and D.-Q. D. designed the study. J.-P. P., C.-D. Z., X. F., Y. B., S. Y. and Z.-M. Z. collected the data. All authors reviewed the manuscript.

\section{Competing Interests}

The authors have declared that no competing interest exists.

\section{References}

1. Siegel RL, Miller KD, Jemal A. Cancer statistics, 2020. CA: a cancer journal for clinicians. 2020; 70(1): 7-30.

2. Greene FL. Current TNM staging of colorectal cancer. The lancet oncology. 2007:8(7):572-573.

3. Edge SB, Byrd DR, Compton CC, Fritz AG, Greene FL. AJCC Cancer Staging Manual. 7th ed. New York, NY: Springer-Verlag. 2010.

4. Amin MB, Edge S, Greene F, et al. AJCC cancer staging manual. 8th ed. New York, NY: Springer-Verlag. 2017.

5. Hashiguchi $Y$, Hase K, Kotake K, et al. Evaluation of the seventh edition of the tumour, node, metastasis (TNM) classification for colon cancer in two nationwide registries of the United States and Japan. Colorectal disease. 2012;14(9):1065-1074.

6. Chu QD, Zhou M, Medeiros KL, Peddi P. Positive surgical margins contribute to the survival paradox between patients with stage IIB/C (T4N0) and stage IIIA (T1-2N1, T1N2a) colon cancer. Surgery. 2016;160(5):1333-1343.

7. Chu QD, Zhou M, Medeiros KL, Peddi P, Kavanaugh M, Wu XC . Poor survival in stage IIB/C (T4N0) compared to stage IIIA (T1-2 N1, T1N2a) colon cancer persists even after adjusting for adequate lymph nodes retrieved and receipt of adjuvant chemotherapy. BMC cancer. 2016;16:460.

8. Huang B, Mo S, Zhu L, Xu T, Cai G. The survival and clinicopathological differences between patients with stage IIIA and stage II rectal cancer: An analysis of 12,036 patients in the SEER database. Oncotarget. 2016;7(48):79787-79796.

9. Kim MJ, Jeong SY, Choi Sj, et al. Survival paradox between stage IIB/C (T4N0) and stage IIIA (T1-2N1) colon cancer. Annals of surgical oncology. 2015;22(2):505-512.

10. Rottoli M, Stocchi L, Dietz DW. T4N0 colon cancer has oncologic outcomes comparable to stage III in a specialized center. Annals of surgical oncology. 2012;19(8):2500-2505

11. Seung KH, Min KK, Byol LS, et al. Clinicopathological and biomolecular characteristics of stage IIB/IIC and stage IIIA colon cancer: Insight into the survival paradox. Journal of surgical oncology. 2019;120(3):423-430.

12. Mazzaferro V, Llovet JM, Miceli R, et al. Predicting survival after liver transplantation in patients with hepatocellular carcinoma beyond the Milan criteria: a retrospective, exploratory analysis. The lancet oncology. 2009;10(1):35-43.

13. Sasaki K, Margonis GA, Andreatos N, et al. The prognostic utility of the "Tumor Burden Score" based on preoperative radiographic features of colorectal liver metastases. Journal of surgical oncology. 2017;116(4):515-523.

14. Kazunari S, Daisuke M, Simone C, et al. The tumor burden score: a new "Metro-ticket" prognostic tool for colorectal liver metastases based on tumor size and number of tumors. Annals of surgical oncology. 2018;267(1):132-141.

15. Lu J, Zheng ZF, Wang W, et al. A novel TNM staging system for gastric Cancer based on the metro-ticket paradigm: a comparative study with the AJCC-TNM staging system. Gastric Cancer. 2019;22(4):759-768.

16. Sahara K, Tsilimigras DI, Mehta R, et al. A novel online prognostic tool to predict long-term survival after liver resection for intrahepatic cholangiocarcinoma: The "metro-ticket" paradigm. Journal of surgical oncology. 2019;120(2):223-230.

17. Akaike H. A new look at the statistical model identification. IEEE Trans on Automatic Control. 1975;19(6):716-723.

18. Carolina AA, Thomas A, Michael W, et al. Discrimination and calibration of clinical prediction models: users' guides to the medical literature. Journal of the American Medical Association. 2017;318(14):1377-1384.

19. Fitzgerald M, Saville BR, Lewis RJ. Decision curve analysis. Journal of the American Medical Association. 2015;313(4):409-410.

20. Hari DM, Leung AM, Lee JH, et al. AJCC cancer staging manual 7th edition criteria for colon cancer: do the complex modifications improve prognostic assessment? Journal of the American College of Surgeons. 2013;217(2):181-190.

21. O'Connell JB, Maggard MA, Ko CY. Colon cancer survival rates with the new American Joint Committee on Cancer sixth edition staging. Journal of the National Cancer Institute. 2004; 19:1420-1425.
22. Edge SB, Sobin LH, Page DL, Gospodarowicz MK, Winchester DP. Re: Colon cancer survival rates with the new American Joint Committee on Cancer sixth edition staging. Journal of the National Cancer Institute. 2005;97(6):463-464.

23. Benson, B. A. American Society of clinical oncology recommendations on adjuvant chemotherapy for stage II colon cancer. Journal of Clinical Oncology. 2004;22(16):3408-3419.

24. Landmann RG, Weiser MR. Surgical management of locally advanced and locally recurrent colon cancer. Clinics in Colon \& Rectal Surgery. 2005;18(03):182-189.

25. Maak M, Simon I, Nitsche U, et al. Independent validation of a prognostic genomic signature (ColoPrint) for patients with stage II colon cancer. Annals of Surgery. 2013;257(6):1053-1058.

26. Webber EM, Lin JS, Whitlock EP. Oncotype DX tumor gene expression profiling in stage II colon cancer. Plos Currents. 2010;2(2):RRN1177.

27. Li J, Guo BC, Sun LR, et al. TNM staging of colorectal cancer should be reconsidered by $\mathrm{T}$ stage weighting. World Journal of Gastroenterology. 2014:20(17):5104-5112.

28. $\mathrm{Li} \mathrm{J}, \mathrm{Yi} \mathrm{CH}, \mathrm{Hu} \mathrm{YT}$, et al. TNM staging of colorectal cancer should be reconsidered according to weighting of the $\mathrm{T}$ stage: verification based on a 25-year follow-up. Medicine. 2016;95(6):e2711.

29. Greene FL, Page DL, Fleming ID, et al. American Joint Committee on Cancer (AJCC) cancer staging manual. 6th ed. New York, NY: Springer-Verlag. 2002.

30. Rice TW, Rusch VW, Ishwaran H, et al. Cancer of the esophagus and esophagogastric junction: data-driven staging for the seventh edition of the American Joint Committee on Cancer/International Union against cancer cancer staging manuals. Cancer. 2010;116(16):3763-3773. 